\title{
Newly Discovered Rope Worm Infections: First Case Report in Gulf Cooperation Council Countries
}

\author{
Ali Sher ${ }^{1 *}$, Saif A. Latif ${ }^{2}$ \\ ${ }^{1}$ Infectious Diseases Hospital Laboratories, Ministry of Health, Kuwait City, Kuwait \\ ${ }^{2}$ North Khaitan Clinic, Ministry of Health, Kuwait City, Kuwait \\ Email: ^alisher02@yahoo.com, saiflatif@hotmail.com
}

How to cite this paper: Sher, A. and Latif, S.A. (2021) Newly Discovered Rope Worm Infections: First Case Report in Gulf Cooperation Council Countries. Open Access Library Journal, 8: e8196.

https://doi.org/10.4236/oalib.1108196

Received: November 17, 2021

Accepted: December 12, 2021

Published: December 15, 2021

Copyright $\odot 2021$ by author(s) and Open Access Library Inc.

This work is licensed under the Creative Commons Attribution International License (CC BY 4.0).

http://creativecommons.org/licenses/by/4.0/

(c) (i) Open Access

\begin{abstract}
Rope worms are anaerobic worms that were discovered in 2009 during the cleansing of the colon by coffee or cleansing enemas. They are rope-like meter-long intestinal parasites and look like twisted fibres of rope attached to the intestinal wall. Thousands of people have passed the rope worms in their stool all over the world. Aim: This study aimed to find the cause of intestinal blockage by the worm passing in stool, identify them and treat the patient. Morphology: The rope worms look like a rope and slimy in nature and over a meter long. They were identified in five different developmental stages based on their morphology. Clinical Symptoms: The rope worm produces a slimy sticky substance with a distinct odour, twists like a corkscrew, and may obstruct the intestine. It may also cause abdominal pain, bloating, gases, indigestion, and constipation. Conclusion: The patients were treated several times in the area clinic with anthelminthic drugs with no improvement of symptoms. The patients were unable to pass the stool without water or milk rectal enemas.
\end{abstract}

\section{Subject Areas \\ Gastroenterology, Infectious Diseases, Parasitic Diseases}

\section{Keywords}

Rope Worm, Enema, Obstruction, Slime

\section{Background}

Humans are hosts to nearly 300 species of helminthic worms and over 70 species of protozoa, some derived from primates and some from animals. Many of these 
are rare and accidental, but humans harbour about 90 common species, which cause very serious diseases. Recently another potential intestinal inhabitant was discovered and given the name rope worm [1] [2] [3]. The rope worms live anaerobically in the human gastro-intestinal tract and were reported in 2009 during the cleansing of the colon by coffee enemas [4]. Their color is often a dark brown, similar to that of feces. When expelled from the body and exposed to air, they produce a distinct and off-putting odour. According to some scientists, they are parasitic worms, but others believe that they are nothing more than a buildup of intestinal mucus and other debris [5]. They are rope-like meter-long intestinal parasites and look like twisted fibres of rope attached to the intestines with suction cups or heads that develop from suction bubbles, twist like a corkscrew, and may obstruct the intestine [6]. Volinsky et al. in 2013 have published and explained the five distinct stages of the rope worm. In the initial stages, the worm consists of slimy mucus and by the fifth and final stage, the worm resembles a viscous mucus rope measuring approximately 1 meter in length [4]. The rope worm uses bubbles for propulsion and movement by creating suction that helps the worm attach to the intestinal wall and preventing it from passing out of the body during defecation. The worm may cause non-specific symptoms, including weight gain or loss, food allergies, common colds, coughing, back pain, rashes, headaches, indigestion, hair loss. They also produce slimy substances with a distinct odour [4].

\section{Case Summary}

\subsection{First Case}

A 57-year-old Egyptian reported to the outpatient clinic of Infectious Diseases Hospital, Kuwait on $16^{\text {th }}$ September 2017, complaining of abdominal pain, bloating, gases, indigestion, and constipation for the last two years. He was unable to pass the stool without water rectal enema. He brought the stool with a worm-like structure after enema and the stool sample was sent to Infectious Diseases Hospital Laboratory for ova and parasites investigation. The macroscopic examination of the stool specimen showed two feet long Taenia-like worm, irregular cylindrical and rope-like shape, slimy in nature with off-putting odour (Figure 1). However, the microscopy of the worm does not show any taenia-like morphological features. All the characters of this worm were very similar to the rope worm reported earlier.
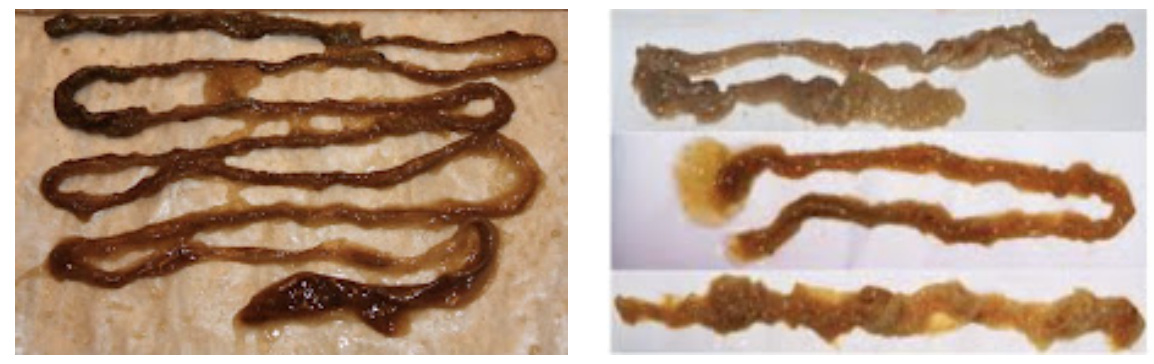

Figure 1. Rope worms identified in stool samples. 


\subsection{Second Case}

A 30-year-old female, Egyptian visited the outpatient clinic of Infectious Diseases Hospital, Kuwait on $14^{\text {th }}$ January 2018 complaining of abdominal pain, bloating, gases, indigestion, constipation, and blockage of intestine for the last one year. She was unable to pass the stool without water rectal enema. She brought the stool with a worm-like structure after enema and the stool sample was sent to Infectious Diseases Hospital Laboratory for ova and parasites investigation. The macroscopic examination of the stool specimen showed adult worm about one feet long and several smaller Taenia-like worm, irregular cylindrical and rope-like shape, slimy in nature with off-putting odour (Figure 1). The morphological features of the worm do not exhibit any taenia-like characters in microscopy. All the characters of this worm were alike to the rope worm reported before. The patient was treated by physicians from the area clinic with multiple courses of albendazole with no improvement of symptoms and passage of the worm.

\section{Discussion}

Several thousand cases of rope worm infections have been reported from all over the world [4]. The adult stages live in the human gastro-intestinal tract and are anaerobic. The rope worms have been discovered during cleansing enemas [4]. They move inside the body by releasing gas bubbles. According to some scientists, they are parasitic worms due to the presence of human DNA, whereas others believe that they are nothing more than a buildup of intestinal mucus and other debris [4]. The structure of the adult rope worms was identified microscopically that they have multiple microchannels running along their length and filled with gas bubbles. The scanning electron microscopy showed that these microchannels terminated on the surface of the worm [4]. However, the rope worms have not been identified completely for the following reasons: 1) rarely come out as whole fully developed adult species; 2) resemble human excrements; 3) do not move outside the human body; 4) often mistaken for the lining of the intestine. More studies are required to identify, characterize and give a proper place to rope worms in parasitological classification. The rope worms are still falling under the "taxonomy unknown" category and are yet to be elucidated.

\section{Conclusion}

We report two cases of rope worm infection in a 57-year-old male and a 30-yearold female Egyptian patient who were presented with vague abdominal pain, bloating, gases, indigestion, constipation, and obstruction of the intestine for the last two years. The microscopy of the worm showed the characteristics of a rope worm. The symptoms did not improve after several courses of albendazole treatment. The obstruction due to rope worms was removed using enemas with milk and salt, soda, eucalyptus, followed by the freshly squeezed lemon juice or freshwater. The patients are under our observation and are still passing the rope 
worms. They can't pass the stool without using enemas. It is speculated that the rope parasite is a biofilm composed of different parasite DNA, previously known as simply "mucus". The results of the DNA analysis of rope worms are still inconclusive, and further research is required to identify the rope worms and place them under the appropriate "taxonomy category".

\section{Acknowledgements}

We would like to thank all the technical staff of the hematology and microbiology sections of the IDH laboratories for their technical help.

\section{Funding}

The financial support for this study was provided by the Ministry of Heath, Kuwait.

\section{Conflicts of Interest}

The authors declare no conflicts of interest regarding the publication of this case report.

\section{References}

[1] Gubarev, N.V., Gubarev, A.V., Orlovskaya, L.P., Orlovskaya, G.M. and Pakulina, O.N. (2006) Method of Human Dehilminthation/Sposob Izgnaniya Gelmintov iz Organizma Cheloveka. Russian Federation Patent RU 11,228110.

[2] Gubarev, N.V. Gubarev, A.V., Lebedev, S.A., Orlovskaya, L.P., Orlovskaya, G.M. and Pakulina O.N. (2007) Method of Human Dehilminthation/Sposob Izgnaniya Gelmintov iz Organizma Cheloveka. Russian Federation Patent RU2270688.

[3] Gubarev, N.V. (2009) Helminthes: Known and....Unknown. Special Literature. First Class Publishing, St. Petersburg, ISBN 978-5-903984-08-4.

[4] Volinsky, A.A., Gubarev, N.V., Orlovskaya, G.M. and Marchenko, E.V. (2013) Development Stages of the "Rope" Human Intestinal Parasite. Arxiv.Org.1301.2845. http://arxiv.org/abs/1301.2845

[5] Volinsky, A.A., Gubarev, N.V., Orlovskaya, G.M. and Marchenko, E.V. (2013) Human Anaerobic Intestinal "Rope" Parasites. arXiv:1301.0953,

http://arxiv.org/abs/1301.0953

[6] Gubarev, N.V., Lebedev, S.A., Orlovskaya, L.P. and Pakulina, O.N. (2007) Method of Human Dehilminthation/Sposob Izgnaniya Gelmintov iz Organizma Cheloveka. Russian Federation Patent RU2250111. 